Pacific

Journal of

Mathematics

AN INTRINSIC VOLUME FUNCTIONAL ON ALMOST COMPLEX 6-MANIFOLDS AND NEARLY KÄHLER GEOMETRY

Misha VERBITSKY

Volume $235 \quad$ No. 2

April 2008 


\title{
AN INTRINSIC VOLUME FUNCTIONAL ON ALMOST COMPLEX 6-MANIFOLDS AND NEARLY KÄHLER GEOMETRY
}

\author{
MISHA VERBITSKY
}

\begin{abstract}
Let $(M, I)$ be an almost complex 6-manifold. The obstruction to the integrability of almost complex structure $N: \Lambda^{0,1}(M) \rightarrow \Lambda^{2,0}(M)$ (the so-called Nijenhuis tensor) maps one 3-dimensional bundle to another 3-dimensional bundle. We say that Nijenhuis tensor is nondegenerate if it is an isomorphism. An almost complex manifold $(M, I)$ is called nearly Kähler if it admits a Hermitian form $\omega$ such that $\nabla(\omega)$ is totally antisymmetric, $\nabla$ being the Levi-Civita connection. We show that a nearly Kähler metric on a given almost complex 6-manifold with nondegenerate Nijenhuis tensor is unique (up to a constant). We interpret the nearly Kähler property in terms of $G_{2^{-}}$ geometry and in terms of connections with totally antisymmetric torsion, obtaining a number of equivalent definitions.

We construct a natural diffeomorphism-invariant functional $I \rightarrow \int_{M}$ Vol $_{I}$ on the space of almost complex structures on $M$, similar to the Hitchin functional, and compute its extrema in the following important case. Consider an almost complex structure $I$ with nondegenerate Nijenhuis tensor, admitting a Hermitian connection with totally antisymmetric torsion. We show that the Hitchin-like functional $I \rightarrow \int_{M} V_{I} I_{I}$ has an extremum in $I$ if and only if $(M, I)$ is nearly Kähler.
\end{abstract}

\section{Introduction}

Almost complex manifolds with nondegenerate Nijenhuis tensor. In geometry, two kinds of plane distributions often arise. There are integrable ones: complex structures, foliations, CR-structures. On the other hand, there are "maximally nonintegrable" distributions, such as the contact structures, where the obstruction

MSC2000: 53C15, 53C25.

Keywords: nearly Kähler, Gray manifold, Hitchin functional, Calabi-Yau, almost complex structure.

Verbitsky is an EPSRC advanced fellow supported by CRDF grant RM1-2354-MO02 and EPSRC grant GR/R77773/01. 
to integrability is nowhere degenerate. Looking at almost complex structures in dimension 3, one finds that the obstruction to integrability, the so-called Nijenhuis tensor

$$
N: \Lambda^{2} T^{1,0}(M) \rightarrow T^{0,1}(M),
$$

maps one 3-dimensional bundle to another 3-dimensional bundle. It is only natural to study the class of complex 3-manifolds such that $N$ is nowhere degenerate.

Given such a manifold $M$, it is possible to construct a nowhere degenerate, positive volume form $\operatorname{det} N^{*} \otimes \overline{\operatorname{det} N^{*}}$ on $M$ (for details, see (1-2)).

We study the extrema of this volume form, showing that these extrema correspond to an interesting geometric structure; see Theorem 2.2.

In Hermitian geometry, one often encounters a special kind of almost complex Hermitian manifolds, called strictly nearly Kähler (NK-)manifolds, or Gray manifolds, after Alfred Gray; see Definition 4.1. These manifolds can be characterized in terms of the $G_{2}$-structure on their Riemannian cone, or in terms of a special set of equations reminiscent of Calabi-Yau equations; see Section 4D.

We prove that a strictly nearly Kähler 3-manifold is uniquely determined by its almost complex structure; see Corollary 3.3. Moreover, such manifolds are extrema of the volume functional associated with the Nijenhuis tensor; see Theorem 2.2. This reminds of the construction of Hitchin's functional on the space of all $S L(3, \mathbb{C})$-structures on a manifold, having extrema on Calabi-Yau manifolds [Hitchin 2000].

This paper has the following structure. In Section 1, we introduce the class of 3-manifolds with nowhere degenerate Nijenhuis tensor, and describe the basic structures associated with these manifolds. We give a sketch of a proof of the existence of a Hermitian connection with totally antisymmetric torsion, due to Friedrich and Ivanov, and show that such a Hermitian metric is uniquely determined by the almost complex structure, if the Nijenhuis tensor is nowhere degenerate.

In Section 2, we introduce the nearly Kähler manifolds, giving several versions of their definition and listing some examples.

In Section 3, we apply the results about connections with totally antisymmetric torsion to nearly Kähler geometry, showing that an almost complex structure determines the Hermitian structure on such a manifold uniquely, up to a constant multiplier.

In Section 4, we give several additional versions of the definition of a nearly Kähler manifold, obtaining an explicit description of a Nijenhuis tensor in terms of an orthonormal frame. We also interpret the nearly Kähler structure on a manifold in terms of $G_{2}$-geometry of its Riemannian cone. This is used to show that an NK-structure on a manifold $M$ is uniquely determined by its metric, unless $M$ is locally isometric to a 6-sphere; see Proposition 4.7. 
In Section 5, we study infinitesimal variations of an almost complex structure. We prove that NK-manifolds are extrema of an intrinsic volume functional described earlier. A partial converse result is also obtained. Given an almost complex manifold $M$ with nowhere degenerate Nijenhuis tensor, admitting a Hermitian connection with totally antisymmetric torsion, $M$ is an extremum of the intrinsic volume functional if and only if $M$ is nearly Kähler.

\section{Almost complex manifolds with nondegenerate Nijenhuis tensor}

1A. Nijenhuis tensor on 6-manifolds. Let $(M, I)$ be an almost complex manifold. The Nijenhuis tensor maps two $(1,0)$-vector fields to the $(0,1)$-part of their commutator. This map is $C^{\infty}$-linear, and vanishes, as the Newlander-Nirenberg theorem implies, precisely when $I$ is integrable. We write the Nijenhuis tensor as

$$
N: \Lambda^{2} T^{1,0}(M) \rightarrow T^{0,1}(M) .
$$

The dual map

$$
N^{*}: \Lambda^{0,1}(M) \rightarrow \Lambda^{2,0}(M)
$$

is also called the Nijenhuis tensor. Cartan's formula implies that $N^{*}$ acts on $\Lambda^{1}(M)$ as the $(2,-1)$-part of the de Rham differential.

When one studies the distributions, one is usually interested in integrable ones (such as $T^{1,0}(M) \subset T M \otimes \mathbb{C}$ for complex or CR-manifolds) or ones where the obstruction to integrability is nowhere degenerate (such as a contact distribution).

For the Nijenhuis tensor in complex dimension $>3$, nondegeneracy does not make much sense, because the space $\operatorname{Hom}\left(\Lambda^{0,1}(M), \Lambda^{2,0}(M)\right)$ becomes quite complicated. However, for $n=3$, both sides of (1-1) are 3-dimensional, and we can define the nondegeneracy as follows.

Definition 1.1. Let $(M, I)$ be an almost complex manifold of real dimension 6, and $N: \Lambda^{2} T^{1,0}(M) \rightarrow T^{0,1}(M)$ the Nijenhuis tensor. We say that $N$ is nondegenerate if $N$ is an isomorphism everywhere. Then $(M, I)$ is called an almost complex 6-manifold with nowhere degenerate Nijenhuis tensor.

Remark 1.2. Such manifolds were investigated by R. Bryant. His results were presented at a conference [Bryant 2000], but never published. The present author unfortunately did not attend the conference and was not aware of his work.

The first thing one notices is that the determinant $\operatorname{det} N^{*}$ gives a section

$$
\operatorname{det} N^{*} \in \Lambda^{3,0}(M)^{\otimes 2} \otimes \Lambda^{3,0}(M)^{*} .
$$

Taking

$$
\operatorname{det} N^{*} \otimes \overline{\operatorname{det} N^{*}} \in \Lambda^{3,0}(M) \otimes \Lambda^{0,3}(M)=\Lambda^{6}(M),
$$


we obtain a nowhere degenerate real volume form $\mathrm{Vol}_{I}$ on $M$. This form is called the canonical volume form associated with the Nijenhuis tensor. This gives a functional $\Psi: I \rightarrow \int_{M} \mathrm{Vol}_{I}$ on the space of almost complex structures. One of the purposes of this paper is to investigate the critical points of the functional $\Psi$, in the spirit of Hitchin's work [2000; 2001].

1B. Connections with totally antisymmetric torsion. Let $(M, g)$ be a Riemannian manifold, $\nabla: T M \rightarrow T M \otimes \Lambda^{1} M$ a connection, and $T \subset \Lambda^{2} M \otimes T M$ its torsion. Identifying $T M$ and $\Lambda^{1} M$ via $g$, we may consider $T$ as an element in $\Lambda^{2} M \otimes \Lambda^{1} M$, that is, a 3 -form on $T M$. If $T$ is totally skew-symmetric as a 3-form on $T M$, we say that $\nabla$ is a connection with totally skew-symmetric (or totally antisymmetric) torsion. If, in addition, $M$ is Hermitian, and $\nabla$ preserves the Hermitian structure, we say that $\nabla$ is a Hermitian connection with totally antisymmetric torsion.

Connections with totally skew-symmetric torsion are extremely useful in physics and differential geometry. An important example of such a connection is provided by a theorem of Bismut [1989].

Theorem 1.3. Let $(M, I)$ be a complex manifold, and $g$ a Hermitian metric. Then $M$ admits a unique connection with totally skew-symmetric torsion preserving I and $g$.

Connections with totally skew-symmetric torsion were studied at great length by Friedrich, Ivanov and others; see for example [Friedrich and Ivanov 2002; Friedrich 2003; Agricola and Friedrich 2004]. Bismut's theorem requires the base manifold to be complex. Motivated by string theory, Friedrich and Ivanov generalized Bismut's theorem to nonintegrable almost complex manifolds [Friedrich and Ivanov 2002]. For completeness, we sketch a proof of their theorem below.

Theorem 1.4. Let $(M, I, \omega)$ be an almost complex Hermitian manifold, and

$$
N: \Lambda^{2} T^{1,0}(M) \rightarrow T^{0,1}(M)
$$

the Nijenhuis tensor. Consider the 3-linear form

$$
\begin{aligned}
& \rho: T^{1,0}(M) \times T^{1,0}(M) \times T^{1,0}(M) \rightarrow \mathbb{C}, \\
& \rho(x, y, z):=\omega(N(x, y), z)
\end{aligned}
$$

Then $M$ admits a connection $\nabla$ with totally skew-symmetric torsion preserving $(\omega, I)$ if and only if $\rho$ is skew-symmetric. Moreover, such a connection is unique.

Sketch of a proof. Theorem 1.4 is proven essentially in the same way as one proves Bismut's theorem and the existence and uniqueness of a Levi-Civita connection. Let $(M, I, g)$ be a Hermitian manifold, and $\nabla_{0}$ a Hermitian connection. Then all Hermitian connections can be obtained by taking $\nabla(A):=\nabla_{0}+A$, where $A$ is 
a 1-form with coefficients in the algebra $\mathfrak{u}(T M)$ of all skew-Hermitian endomorphisms. The torsion $T_{A}$ of $\nabla(A)$ is written as

$$
T_{A}=T_{0}+\operatorname{Alt}_{12}(A),
$$

where $T_{0}$ is a torsion of $\nabla_{0}$, and Alt 12 denotes the antisymmetrization of

$$
\Lambda^{1}(M) \otimes \mathfrak{u}(T M) \subset \Lambda^{1}(M) \otimes \Lambda^{1}(M) \otimes T M
$$

over the first two indices. We identify $\mathfrak{u}(T M)$ with $\Lambda^{1,1}(M)$ in a standard way. Then Theorem 1.4 can be reinterpreted as a statement about linear-algebraic properties of the operator

$$
\operatorname{Alt}_{12}: \Lambda^{1}(M) \otimes \Lambda^{1,1}(M) \rightarrow\left(\Lambda^{2}(M) \otimes \Lambda^{1}(M)\right)^{(2,1)+(1,2)}
$$

(where the superscript $(\ldots)^{(2,1)+(1,2)}$ means taking $(2,1)+(1,2)$-part with respect to the Hodge decomposition), as follows.

By definition, the Nijenhuis tensor $N$ is a section of $\Lambda^{2,0} \otimes T^{0,1}$. Identifying $T^{0,1}$ with $\Lambda^{1,0}$ via $g$, we can consider $N$ as an element of $\Lambda^{2,0} \otimes \Lambda^{1,0}$. By Cartan's formula, $N$ is equal to the $(3,0)$-part of the torsion. Therefore, the existence of a connection with totally skew-symmetric torsion implies that (1-3) is skewsymmetric.

Conversely, assume that (1-3) is skew-symmetric. Since (1-4) maps $\Lambda^{1}(M) \otimes$ $\Lambda^{1,1}(M)$ to $(2,1) \oplus(1,2)$-tensors, the $(3,0)$ and $(0,3)$-parts of torsion stay skewsymmetric if we modify the connection by adding $A \in \Lambda^{1} \otimes \mathfrak{u}(T M)$. Denote by $T_{1}$ the $(2,1) \oplus(1,2)$-part of the torsion $T_{0}$. To prove Theorem 1.4, we need to find $A \in \Lambda^{1}(M) \otimes \Lambda^{1,1}(M)$ such that $T_{1}-\operatorname{Alt}_{12}(A)$ is totally skew-symmetric.

The map $\operatorname{Alt}_{12}: \Lambda^{1}(M) \otimes \Lambda^{2}(M) \rightarrow \Lambda^{2}(M) \otimes \Lambda^{1}(M)$ is an isomorphism, as a dimension count implies (this map has no kernel, which is easy to see). Therefore, (1-4) is injective. Using the dimension count again, we find that cokernel of (1-4) projects isomorphically into

$$
\Lambda^{2,1}(M) \oplus \Lambda^{1,2}(M) \subset \Lambda^{2}(M) \otimes \Lambda^{1}(M) .
$$

Therefore, for any $T_{1}$ in $(2,1) \oplus(1,2)$-part of $\Lambda^{2}(M) \otimes \Lambda^{1}(M)$ there exists $A \in$ $\Lambda^{1}(M) \otimes \Lambda^{1,1}(M)$ and $B \in \Lambda^{2,1}(M) \oplus \Lambda^{1,2}(M)$ such that $T_{1}=\operatorname{Alt}_{12}(A)+B$.

\section{C. Connections with antisymmetric torsion on almost complex 6-manifolds.}

Let $(M, I)$ be an almost complex manifold, $N$ its Nijenhuis tensor. To obtain all Hermitian connections with totally skew-symmetric torsion on $(M, I)$, one needs to find all metrics $g$ for which the tensor $\omega(N(x, y), z)$ is skew-symmetric. As Theorem 1.4 implies, these metrics are precisely those for which such a connection exists.

We also prove the following proposition. 
Proposition 1.5. Let $(M, I)$ be an almost complex 6-manifold with Nijenhuis tensor which is nondegenerate in a dense subset of $M$, and $g$ a Hermitian metric admitting a connection with totally antisymmetric torsion. Then $g$ is uniquely determined by $I$, up to conformal equivalence. Moreover, the Riemannian metric $g$ determines I uniquely, unless $(M, g)$ is locally isometric to a 6-sphere.

Proof. This is Proposition 3.1 and Proposition 4.7.

1D. Correspondence with the results of $R$. Bryant. Since the first version of this paper was written, the previously unpublished results of R. Bryant appeared in a fundamental and important preprint [2006]. There is a significant overlap with our research, though the presentation and terminology are different. The property (1-3) (which is equivalent to the existence of Hermitian connection with totally antisymmetric curvature) is called "Nijenhuis tensor of real type" in [Bryant 2006]. The main focus of that preprint is the so-called "quasiintegrable almost complex manifold": manifolds with Nijenhuis tensor of real type, which is at every point of $M$ either nondegenerate (of constant signature) or zero. Examples of such structures are found. In particular, all twistor spaces of Kähler surfaces with sign-definite holomorphic bisectional curvature are shown to be quasiintegrable. A variant of Theorem 2.2 is also proven. It is shown that nearly Kähler manifolds are critical points of the functional $\mathrm{Vol}_{I}$ [Bryant 2006, Proposition 8].

\section{Nearly Kähler manifolds: an introduction}

Nearly Kähler manifolds (also known as $K$-spaces or almost Tachibana spaces) were defined and studied by Alfred Gray in $[1965 ; 1970 ; 1971 ; 1976]$ in a general context of intrinsic torsion of $U(n)$-structures and weak holonomies. An almost complex Hermitian manifold $(M, I)$ is called nearly Kähler if $\nabla_{X}(I) X=0$, for any vector fields $X$ ( $\nabla$ denotes the Levi-Civita connection). In other words, the tensor $\nabla \omega$ must be totally skew-symmetric, for $\omega$ the Hermitian form on $M$. If $\nabla_{X}(\omega) \neq 0$ for any nonzero vector field $X, M$ is called strictly nearly Kähler.

In this section, we give an overview of known results and "folk theorems" of nearly Kähler geometry. Most of this theory was known (in a different context) since 1980s, when the study of Killing spinors was initiated [Baum et al. 1991].

2A. Splitting theorems for nearly Kähler manifolds. As V. F. Kirichenko proved, nearly Kähler manifolds admit a connection with totally antisymmetric, parallel torsion [Kiričenko 1977]. This observation was used to prove a splitting theorem for nearly Kähler manifolds: any nearly Kähler manifold is locally a Riemannian product of a Kähler manifold and a strictly nearly Kähler one [Gray 1976; Nagy 2002b]. 
A powerful classification theorem for Riemannian manifolds admitting an orthogonal connection with irreducible connection and parallel torsion was obtained by R. Cleyton and A. Swann [2004]. They proved that any such manifold either is locally homogeneous, or has vanishing torsion, or has weak holonomy $G_{2}$ (in dimension 7) or $S U$ (3) (in dimension 6).

Using Kirichenko theorem, this result can be used to obtain a classification of nearly Kähler manifolds. P.-A. Nagy has shown [2002a] that any strictly nearly Kähler manifold is locally a product of locally homogeneous manifolds, strictly nearly Kähler 6-manifolds, and twistor spaces of quaternionic Kähler manifolds of positive Ricci curvature, equipped with the Eells-Salamon metric.

These days the term "nearly Kähler" usually denotes strictly nearly Kähler 6manifolds. In sequel we shall follow this usage, often omitting "strictly" and "6dimensional".

In dimension 6, a manifold is (strictly) nearly Kähler if and only if it admits a Killing spinor [Grunewald 1990]. Therefore, such a manifold is Einstein, with positive Einstein constant.

As one can easily show (see Theorem 4.2), strictly nearly Kähler 6-manifolds can be defined as 6-manifolds with structure group $S U(3)$ and fundamental forms $\omega \in \Lambda_{\mathbb{R}}^{1,1}(M), \Omega \in \Lambda^{3,0}(M)$, satisfying $d \omega=3 \lambda \operatorname{Re} \Omega$ and $d \operatorname{Im} \Omega=-2 \lambda \omega^{2}$. An excellent introduction to nearly Kähler geometry is found in [Moroianu et al. 2005].

The most puzzling aspect of nearly Kähler geometry is a complete lack of nonhomogeneous examples. With the exception of four homogeneous cases described below (Section 2C), no other compact examples of strictly nearly Kähler 6-manifolds are known to exist.

2B. Nearly Kähler manifolds in $G_{2}$-geometry and physics. Nearly Kähler manifolds have many uses in geometry and physics. Along with Calabi-Yau manifolds, nearly Kähler manifolds appear as target spaces for supersymmetric sigma-models, solving equations of type II string theory. These manifolds are the only 6-manifolds admitting a Killing spinor. This implies that a Riemannian cone $C(M)$ of a nearly Kähler manifold has a parallel spinor.

Let $(M, g)$ be a Riemannian manifold. Recall that the Riemannian cone of $(M, g)$ is a product $M \times \mathbb{R}^{>0}$, with a metric $g t^{2} \oplus \lambda \cdot d t^{2}$, where $t$ is a unit parameter on $\mathbb{R}^{>0}$, and $\lambda$ a constant. It is well known that $M$ admits a real Killing spinor if and only if $C(M)$ admits a parallel spinor (for appropriate choice of $\lambda$ ). Then, $C(M)$ has restricted holonomy, for any nearly Kähler 6-manifold. It is easy to check that in fact $C(M)$ has holonomy $G_{2}$. This explains a tremendous importance that nearly Kähler manifolds play in $G_{2}$-geometry.

We give a brief introduction of $G_{2}$-geometry, following [Hitchin 2000] and [Joyce 2000]. Let $V^{7}$ be a 7-dimensional real vector space. The group $G L(7, \mathbb{R})$ 
acts on $\Lambda^{3}\left(V^{7}\right)$ with two open orbits. For $v$ in one of these orbits, its stabilizer $S t(v) \subset G L(7, \mathbb{R})$ is 14 -dimensional, as a dimension count insures. It is easy to check that $S t(v)$ is a real form of a Lie group $G_{2}$. For one of these orbits, $S t(v)$ is a compact form of $G_{2}$, for another one it is noncompact. A 3-form $v \in \Lambda^{3}\left(V^{7}\right)$ is called stable if its stabilizer is a compact form of $G_{2}$.

A 7-manifold $X$ equipped with a 3 -form $\rho$ is called a $G_{2}$-manifold if $\rho$ is stable everywhere in $X$. In this case, the structure group of $X$ is reduced to $G_{2}$. Also, $X$ is equipped with a natural Riemannian structure:

$$
\left.\left.x, y \rightarrow \int_{X}(\rho\lrcorner x\right) \wedge(\rho\lrcorner x\right) \wedge \rho \quad(x, y \in T M) .
$$

A $G_{2}$-manifold is called parallel if $\nabla \rho=0$, where $\nabla$ is the Levi-Civita connection associated with this Riemannian structure.

Isolated singularities of $G_{2}$-manifolds are of paramount importance in physics; see [Acharya and Gukov 2004; Atiyah and Witten 2002]. A simplest example of an isolated singular point is a conical singularity.

A metric space $X$ with marked points $x_{1}, \ldots, x_{n}$ is called a space with isolated singularities, if $X \backslash\left\{x_{1}, \ldots, x_{n}\right\}$ is a Riemannian manifold. Consider a space $(X, x)$ with a single singular point. The singularity $x \in X$ is called conical if $X$ is equipped with a flow acting on $X$ by homotheties and contracting $X$ to $x$. In this case, $X \backslash x$ is isomorphic to a Riemannian cone of a Riemannian manifold $M$.

It is easy to check that the cone $C(M)$ of a nearly Kähler manifold is equipped with a parallel $G_{2}$-structure, and, conversely, every conical singularity of a parallel $G_{2}$-manifold is obtained as $C(M)$, for some nearly Kähler manifold $M$ [Hitchin 2001; Ivanov et al. 2006]. For completeness' sake, we give a sketch of a proof of this result in Proposition 4.5.

The idea of this correspondence is quite clear. Let $X=C(M)$ be a parallel $G_{2}$ manifold, and $\omega_{C}$ its 3-form. Unless $X$ is flat, we may assume that $X$ has holonomy which is equal to $G_{2}$ and not its proper subgroup. Indeed, if holonomy of $X$ is less than $G_{2}$, by Berger's classification of irreducible holonomies, $X$ is represented (as a Riemannian manifold) as a product of manifolds of smaller dimension. However, the singular point of the metric completion $\bar{X}$ is isolated, and this precludes such a decomposition, unless $\bar{X}$ is smooth. In the latter case, $X$ is flat.

Since the holonomy of $X$ is (strictly) $G_{2}$, the 3 -form can be reconstructed from the Riemannian structure uniquely. After rescaling, we may assume that the Riemannian structure on $X=C(M)$ is homogeneous of weight 2, with respect to the action of $\mathbb{R}^{>0}$ on $C(M)$. Then $\omega_{C}$ is homogeneous of weight 3 . Homogeneous $G_{2}$-structures on $C(M)$ correspond naturally to $S U$ (3)-structures on $M$. We write $\omega_{C}$ as $t^{2} \pi^{*} \omega \wedge d t+t^{3} \pi^{*} \rho$, where $\rho, \omega$ are forms on $M$, and $\pi: C(M) \rightarrow M$ is the standard projection. From a local coordinate expression of a $G_{2}$-form, we find 
that $\omega$ is a Hermitian form corresponding to an almost complex structure $I$, and $\rho=\operatorname{Re} \Omega$ for a nowhere degenerate $(3,0)$-form $\Omega$ on $(M, I)$.

The converse is proven by the same computation: given an $S U(3)$-manifold $(M, I, \omega, \Omega)$, we write a 3 -form

$$
\omega_{C}:=t^{2} \pi^{*} \omega \wedge d t+t^{3} \pi^{*} \rho,
$$

on $C(M)$, and show that it is a $G_{2}$-structure, using a coordinate expression for a $G_{2}$-form.

As Fernandez and Gray proved in [1982], a $G_{2}$-manifold $\left(X, \omega_{C}\right)$ is parallel if and only if $\omega_{C}$ is harmonic. For the form (2-2), $d \omega_{C}=0$ is translated into $d \omega=3 \rho$.

Since $* \rho=I \rho$ and $* \omega=\omega^{2}$, the condition $d^{*} \omega_{C}=0$ becomes $d I \rho=-2 \omega^{2}$. After an appropriate rescaling, we find that this is precisely the condition defining the nearly Kähler structure Theorem 4.2. Therefore, $C(M)$ is a $G_{2}$-manifold if and only if $M$ is nearly Kähler (see Section $4 \mathrm{C}$ for a more detailed argument).

The correspondence between conical singularities of $G_{2}$-manifolds and nearly Kähler geometry can be used further to study the locally conformally parallel $G_{2^{-}}$ manifolds (see also [Ivanov et al. 2006]). A locally conformally parallel $G_{2}$-manifold is a 7-manifold $M$ with a covering $\tilde{M}$ equipped with a parallel $G_{2}$-structure, with the deck transform acting on $\tilde{M}$ by homotheties. Since homotheties preserve the Levi-Civita connection $\tilde{\nabla}$ on $\tilde{M}, \tilde{\nabla}$ descends to a torsion-free connection on $M$, which is no longer orthogonal, but preserves the conformal class of a metric. Such a connection is called a Weyl connection, and a conformal manifold of dimension $>2$ equipped with a torsion-free connection preserving the conformal class is called a Weyl manifold. The Weyl manifolds are a subject of much study in conformal geometry; see for example [Dragomir and Ornea 1998] and the reference therein.

The key theorem of Weyl geometry is proven by P. Gauduchon [1984]. He has shown that any compact Weyl manifold is equipped with a privileged metric in its conformal class. This metric (called a Gauduchon metric now) is defined as follows.

Let $(M,[g], \nabla)$ be a compact Weyl manifold, where $[g]$ is a conformal class, and $g \in[g]$ any metric within this conformal class. Since $\nabla[g]=0$, we have $\nabla(g)=g \otimes \theta$, where $\theta$ is a 1 -form, called a Lee form. A metric $g$ is called Gauduchon if $\theta$ satisfies $d^{*} \theta=0$. A Gauduchon metric is unique (up to a complex multiplier).

Let now $(M, \nabla,[g])$ be a Weyl manifold with a Ricci-flat connection $\nabla$. In [1995], Gauduchon has shown that the Lee form $\theta$ of the Gauduchon metric on $M$ is parallel with respect to the Levi-Civita connection associated with this metric.

Applying this argument to a compact locally conformally parallel $G_{2}$-manifold $M$, we obtain that the Lee form is parallel. From this one infers that the parallel 
$G_{2}$-covering $\tilde{M}$ of $M$ is a cone over some Riemannian manifold $S$; see for example [Verbitskiı 2004, Proposition 11.1], also see [Kamishima and Ornea 2005] and [Gini et al. 2005]. Using the argument stated above, we find that this manifold is in fact nearly Kähler. Therefore, $S$ is Einstein, with positive Ricci curvature. Since $M$ is compact, $S$ is complete, and by Myers theorem, $S$ is actually compact; see [Verbitskiı 2004, Remark 10.7]. Now, the argument which proves Theorem 12.1 of [Verbitskiu 2004] can be used to show that $\operatorname{dim} H^{1}(M, \mathbb{Q})=1$, and $M=C(S) / \mathbb{Z}$. This gives the following structure theorem, which is proven independently in [Ivanov et al. 2006].

Theorem 2.1. Let $M$ be a compact locally conformally parallel $G_{2}$-manifold. Then $M=C(S) / \mathbb{Z}$, where $S$ is a nearly Kähler manifold, and the $\mathbb{Z}$-action on $C(S) \cong S \times \mathbb{R}^{>0}$ is generated by a map $(x, t) \mapsto(\varphi(x), q t)$, where $|q|>1$ is a real number, and $\varphi: S \rightarrow S$ an automorphism of nearly Kähler structure.

2C. Examples of nearly Kähler manifolds. Just as the conical singularities of parallel $G_{2}$-manifolds correspond to nearly Kähler manifolds, the conical singularities of $\operatorname{Spin}(7)$-manifolds correspond to the so-called "nearly parallel" $G_{2}$-manifolds (see [Ivanov 2004]). A $G_{2}$-manifold $(M, \omega)$ is called nearly parallel if $d \omega=c * \omega$, where $c$ is some constant. The analogy between nearly Kähler 6-manifolds and nearly parallel $G_{2}$-manifolds is almost perfect. These manifolds admit a connection with totally antisymmetric torsion and have weak holonomy $S U(3)$ and $G_{2}$ respectively. N. Hitchin realized nearly Kähler 6-manifolds and nearly parallel $G_{2}-$ manifolds as extrema of a certain functional, called Hitchin functional by physicists (see [Hitchin 2001]).

However, examples of nearly parallel $G_{2}$-manifolds are found in profusion (every 3-Sasakian manifold is nearly parallel $G_{2}$ ), and compact nearly Kähler manifolds are rare.

Only four compact examples are known (see the list below); all of them homogeneous. In [Butruille 2005] it was shown that any homogeneous nearly Kähler 6-manifold belongs to this list.

(1) The 6-dimensional sphere $S^{6}$. Since the cone $C\left(S^{6}\right)$ is flat, $S^{6}$ is a nearly Kähler manifold, as shown in Section 2B. The almost complex structure on $S^{6}$ is reconstructed from the octonion action, and the metric is standard.

(2) $S^{3} \times S^{3}$, with the complex structure mapping $\xi_{i}$ to $\xi_{i}^{\prime}, \xi_{i}^{\prime}$ to $-\xi_{i}$, where $\xi_{i}, \xi_{i}^{\prime}$, $i=1,2,3$ is a basis of left invariant 1 -forms on the first and the second component.

(3) Given a selfdual Einstein Riemannian 4-manifold $M$ with positive Einstein constant, one defines its twistor space $\operatorname{Tw}(M)$ as a total space of a bundle of unit 
spheres in $\Lambda_{-}^{2}(M)$ of antiselfdual 2-forms. Then $\operatorname{Tw}(M)$ has a natural KählerEinstein structure $\left(I_{+}, g\right)$, obtained by interpreting unit vectors in $\Lambda_{-}^{2}(M)$ as complex structure operators on $T M$. Changing the sign of $I_{+}$on $T M$, we obtain an almost complex structure $I_{-}$which is also compatible with the metric $g$ [Eells and Salamon 1985]. A straightforward computation insures that $\left(\operatorname{Tw}(M), I_{-}, g\right)$ is nearly Kähler [Muškarov 1987].

As N. Hitchin proved [1981], there are only two compact selfdual Einstein 4manifolds: $S^{4}$ and $\mathbb{C} P^{2}$. The corresponding twistor spaces are $\mathbb{C} P^{3}$ and the flag space $F(1,2)$. The almost complex structure operator $I_{-}$induces a nearly Kähler structure on these two symmetric spaces.

2D. Nearly Kähler manifolds are extrema of volume on almost complex manifolds with nowhere degenerate Nijenhuis tensor. Let $(M, I, \omega)$ be a nearly Kähler manifold, and $N^{*}: \Lambda^{0,1}(M) \rightarrow \Lambda^{2,0}(M)$ the Nijenhuis tensor. By Cartan's formula, $N^{*}$ is the $(2,-1)$-part of the de Rham differential (with respect to the Hodge decomposition). In Theorem 4.2, it is shown that $d \omega$ is a real part of a nowhere degenerate $(3,0)$-form $\Omega$. Therefore, the 3-form

$$
\omega(N(x, y), z)=d \omega(x, y, z)=\operatorname{Re} \Omega(x, y, z)
$$

is nowhere degenerate on $T^{1,0}(M)$. So the Nijenhuis tensor $N$ is nowhere degenerate.

The main result of this paper is the following theorem, which is analogous to [Hitchin 2001].

Theorem 2.2. Let $(M, I)$ be a compact almost complex 6-manifold with nowhere degenerate Nijenhuis tensor admitting a Hermitian connection with totally antisymmetric torsion. Consider the functional $I \rightarrow \int_{M} \mathrm{Vol}_{I}$ on the space of such manifolds constructed in Section 1A. Then this functional has a critical point at I if and only if $(M, I)$ admits a nearly Kähler metric.

Proof. This follows from Proposition 5.6 and Theorem 4.2.

Remark 2.3. As follows from Corollary 3.3 , the nearly Kähler metric on $(M, I)$ is uniquely determined by the almost complex structure.

\section{Almost complex structures and connections with totally antisymmetric torsion}

Let $(M, I)$ be a 6-dimensional almost complex manifold, and

$$
N^{*}: \Lambda^{0,1}(M) \rightarrow \Lambda^{2,0}(M)
$$

its Nijenhuis tensor. Given a point $x \in M$, the operator $\left.N^{*}\right|_{\Lambda_{x}^{0,1}(M)}$ can a priori take any value within $\operatorname{Hom}\left(\Lambda^{0,1}(M), \Lambda^{2,0}(M)\right)$. For $\left.N^{*}\right|_{\Lambda_{x}^{0,1}(M)}$ generic, the stabilizer 
$\operatorname{St}\left(N_{x}^{*}\right)$ of $N_{x}^{*}$ within $G L\left(T_{x} M\right)$ is 2-dimensional. If we fix a complex parameter, the eigenspaces of $N_{x}^{*}$ (taken in appropriate sense) define a frame in $T M$. Thus, a geometry of a "very generic" 6-dimensional almost complex manifold is rather trivial.

However, for a $N_{x}^{*}$ inside a 10-dimensional subspace

$$
W_{0} \subset \operatorname{Hom}\left(\Lambda^{0,1}(M), \Lambda^{2,0}(M)\right)
$$

(see Remark 3.2), the stabilizer $\operatorname{St}\left(N_{x}^{*}\right)$ contains $S U(3)$, and the geometry of $(M, I)$ becomes more interesting.

Proposition 3.1. Let $(M, I)$ be an almost complex 6-manifold with Nijenhuis tensor which is nondegenerate in a dense set. Assume that $(M, I)$ admits a Hermitian structure $\omega$ and a Hermitian connection with totally antisymmetric torsion. Then $\omega$ is uniquely determined by $I$, up to conformal equivalence.

Proof. Consider the map

$$
C:=\operatorname{Id} \otimes N^{*}: \Lambda^{1,1}(M) \rightarrow \Lambda^{1,0}(M) \otimes \Lambda^{2,0}(M)
$$

obtained by acting with the Nijenhuis tensor $N^{*}: \Lambda^{0,1}(M) \rightarrow \Lambda^{2,0}(M)$ on the second tensor multiplier of $\Lambda^{1,1}(M) \cong \Lambda^{1,0}(M) \otimes \Lambda^{0,1}(M)$. Then $C$ maps $\omega$ to a 3-form $x, y, z \mapsto \omega(N(x, y), z)$. As Theorem 1.4 implies, $(M, I, \omega)$ admits a Hermitian connection with totally antisymmetric torsion if and only if $C(\omega)$ lies inside a 1-dimensional space

$$
\Lambda^{3,0}(M) \subset \Lambda^{1,0}(M) \otimes \Lambda^{2,0}(M) .
$$

However, $C$ is an isomorphism in a dense subset of $M$; hence, all $\omega$ which satisfy the conditions of Theorem 1.4 are proportional.

Remark 3.2. The same argument can be used to prove that an almost complex manifold admits a Hermitian connection with totally antisymmetric torsion if and only if $C^{-1}\left(\Lambda^{3,0}(M)\right)$ contains a Hermitian form. This is the space $W_{0}$ alluded to in the beginning of this section.

Proposition 3.1 leads to the following corollary.

Corollary 3.3. Let $(M, I)$ be an almost complex 6-manifold. Then $(M, I)$ admits at most one strictly nearly Kähler metric, up to a constant multiplier.

Proof. Let $\omega_{1}$ and $\omega_{2}$ be nearly Kähler metrics on $(M, I)$. Since $\left(M, I, \omega_{i}\right)$ is strictly nearly Kähler, the 3-form $C\left(\omega_{i}\right) \in \Lambda^{3,0}(M)$ is nowhere degenerate; see (3-1). Therefore, $(M, I)$ has nowhere degenerate Nijenhuis tensor. Then, by Proposition 3.1, $\omega_{i}$ are proportional: $\omega_{1}=f \omega_{2}$. However, $d \omega_{i}^{2}=0$ on any nearly Kähler 3-manifold; see for example Theorem 4.2 (ii). Then $2 f d f \wedge \omega_{2}^{2}=0$. This implies $d f=0$, because the map $\eta \mapsto \eta \wedge \omega_{2}^{2}$ is an isomorphism on $\Lambda^{1}(M)$. 
Remark 3.4. The converse is also true: unless $(M, g)$ is locally isometric to a 6-sphere, the Riemannian metric $g$ determines the nearly Kähler almost complex structure $I$ uniquely; see Proposition 4.7.

\section{Nearly Kähler geometry and Hermitian connections with totally antisymmetric torsion}

4A. Hermitian structure on $\Lambda^{\mathbf{3 , 0}}(M)$ and nearly Kähler manifolds. Let $(M, I)$ be an almost complex 6-manifold, and $\Omega \in \Lambda^{3,0}(M)$ a nondegenerate $(3,0)$-form. Then $\Omega \wedge \bar{\Omega}$ is a positive volume form on $M$. This gives a $\operatorname{Vol}(M)$-valued Hermitian structure on $\Lambda^{3,0}(M)$. If $M$ is in addition Hermitian, then $M$ is equipped with a natural volume form $\mathrm{Vol}_{h}$ associated with the metric, and the map

$$
\Omega \mapsto \frac{\Omega \wedge \bar{\Omega}}{\operatorname{Vol}_{h}}
$$

can be considered as a Hermitian metric on $\Lambda^{3,0}(M)$. This metric agrees with the usual Riemann-Hodge pairing known from algebraic geometry, when $I$ is integrable. The following definition is a restatement of the classical one; see Section 2.

Definition 4.1. Let $(M, I, \omega)$ be an almost complex Hermitian manifold, and $\nabla$ the Levi-Civita connection. Then $(M, I, \omega)$ is called nearly Kähler if the tensor $\nabla \omega$ is totally antisymmetric, that is, $\nabla \omega \subset \Lambda^{3}(M)$.

The following theorem is a main result of this section.

Theorem 4.2. Let $(M, I, \omega)$ be an almost complex Hermitian 6-manifold equipped with a $(3,0)$-form $\Omega$. Assume that $\Omega$ satisfies $3 \lambda \operatorname{Re} \Omega=d \omega$, and $|\Omega|_{\omega}=1$, where $\lambda$ is a constant, and $|\cdot|_{\omega}$ is the Hermitian metric on $\Lambda^{3,0}(M)$ constructed above. Then the following conditions are equivalent.

(i) $M$ admits a Hermitian connection with totally antisymmetric torsion.

(ii) $d \Omega=-2 \sqrt{-1} \lambda \omega^{2}$.

(iii) $(M, I, \omega)$ is nearly Kähler, and $d \omega=\nabla \omega$.

The equivalence of (ii) and (iii) is known; see for example the second part of the proof of Theorem 6 in [Hitchin 2001].

The existence of Hermitian connections with totally antisymmetric torsion on nearly Kähler manifolds is also well known (see Section 2). This connection is written as $\nabla_{N K}=\nabla+T$, where $\nabla$ is the Levi-Civita connection on $M$, and $T$ the operator obtained from the 3-form $3 \lambda \operatorname{Im} \Omega$ by raising one of the indices. The torsion of $\nabla_{N K}$ is totally antisymmetric by construction (it is equal $T$ ). Also by construction, we find that $T(\omega)=-3 \lambda \operatorname{Re} \Omega$, hence $\nabla_{N K}(\omega)=0$. Therefore, $\nabla_{N K}$ is a Hermitian connection with totally antisymmetric torsion. This takes care of the implication (iii) $\Rightarrow$ (i). 
To prove Theorem 4.2, it remains to prove that (i) implies (ii); we do that in Section 4B. For completeness' sake, we sketch the proof of the implication (ii) $\Rightarrow$ (iii) in Section 4D.

Remark 4.3. As Corollary 3.3 shows, a non-Kähler nearly Kähler metric on $M$ is uniquely determined by the almost complex structure $I$.

\section{B. Connections with totally antisymmetric torsion and Nijenhuis tensor.}

Lemma 4.4. In the assumptions of Theorem 4.2, (i) implies (ii).

Proof. Step 1: We show that $d \Omega \in \Lambda^{2,2}(M)$. Were $(M, I)$ integrable, the differential $\overline{d \text { would }}$ have only $(0,1)$ - and $(1,0)$-part with respect to the Hodge decomposition: $d=d^{1,0}+d^{0,1}$. For a general almost complex manifold, $d$ splits into 4 parts:

$$
d=d^{2,-1}+d^{1,0}+d^{0,1}+d^{-1,2} .
$$

This follows immediately from the Leibniz rule. However,

$$
0=d^{2} \omega=d(\Omega+\bar{\Omega})=d \Omega+d \bar{\Omega} .
$$

Since $\Lambda^{p, q}(M)$ vanishes for $p$ or $q>3$, we also have

$$
d \Omega+d \bar{\Omega}=d^{0,1} \Omega+d^{-1,2} \Omega+d^{2,-1} \bar{\Omega}+d^{1,0} \bar{\Omega} .
$$

The four terms on the right hand side of (4-2) have Hodge types $(3,1),(2,2)$, $(2,2)$ and $(1,3)$. Since their sum vanishes by $(4-1)$, we obtain

$$
d^{0,1} \Omega=0, \quad d^{1,0} \bar{\Omega}=0, \quad d^{2,-1} \bar{\Omega}=-d^{-1,2} \Omega .
$$

Then (4-2) gives

$$
d \Omega=-d^{2,-1} \bar{\Omega}=d^{-1,2} \Omega .
$$

Step 2:

$$
\left.d^{2,-1}\right|_{\Lambda^{1,1}(M)}=\wedge \circ \operatorname{Id} \otimes N^{*},
$$

where $N^{*}: \Lambda^{0,1}(M) \rightarrow \Lambda^{2,0}(M)$ is the Nijenhuis tensor,

$$
\mathrm{Id} \otimes N^{*}: \Lambda^{1,1}(M) \rightarrow \Lambda^{2,0}(M) \otimes \Lambda^{1,0}(M)
$$

acts as $N^{*}$ on the second multiplier of $\Lambda^{1,1}(M) \cong \Lambda^{1,0}(M) \otimes \Lambda^{0,1}(M)$, and $\wedge$ denotes the exterior product. (4-4) is immediately implied by the Cartan's formula for the de Rham differential.

Step 3: From the existence of Hermitian connection with totally antisymmetric torsion we obtain that the form

$$
\omega(N(x, y), z): T^{1,0} M \times T^{1,0} M \times T^{1,0} M \rightarrow \mathbb{C}
$$


is totally antisymmetric; see Theorem 1.4. From (4-4) it follows that

$$
\omega(N(x, y), z)=d \omega=3 \lambda \operatorname{Re} \Omega .
$$

Consider an orthonormal frame $d z_{1}, d z_{2}, d z_{3}$ in $\Lambda^{1,0}(M)$, satisfying

$$
\Omega=d z_{1} \wedge d z_{2} \wedge d z_{3}
$$

(such a frame exists because $|\Omega|_{\omega}=1$ ). Then (4-5) gives

$$
N^{*}\left(d \bar{z}_{i}\right)=\lambda d \check{z}_{i},
$$

where $d \check{z}_{1}=d z_{2} \wedge d z_{3}, d \check{z}_{2}=-d z_{1} \wedge d z_{3}, d \check{z}_{3}=d z_{1} \wedge d z_{2}$.

Step 4: Using Cartan's formula as in Step 2, we express $d^{-1,2} \Omega$ through the Nijenhuis tensor. Then (4-3) can be used to write $d \Omega=d^{-1,2} \Omega$ in terms of $N^{*}$. Finally, (4-6) allows us to write $d^{-1,2} \Omega$ in coordinates, yielding $d \Omega=-2 \sqrt{-1} \lambda \omega^{2}$.

\section{C. $G_{2}$-structures on cones of Hermitian 6-manifolds.}

Proposition 4.5. Let $(M, I, \omega)$ be an almost complex Hermitian manifold, $\Omega \in$ $\Lambda^{3,0}(M)$ a (3,0)-form which satisfies $d \omega=3 \lambda \operatorname{Re} \Omega$, for some real constant, and $|\Omega|_{\omega}=1$. Assume, in addition, that $d \Omega=-2 \sqrt{-1} \lambda \omega^{2}$. Consider the cone $C(M)=$ $M \times \mathbb{R}^{>0}$, equipped with a 3-form $\rho=3 t^{2} \omega \wedge d t+t^{3} d \omega$, where $t$ is the unit parameter on the $\mathbb{R}^{>0}$-component. Then $(C(M), \rho)$ is a parallel, $G_{2}$-manifold; see Section $2 B$. Moreover, any parallel $G_{2}$-structure $\rho^{\prime}$ on $C(M)$ is obtained in this way if $\rho^{\prime}$ is homogeneous of weight 3 with respect to the natural action of $\mathbb{R}^{>0}$ on $C(M)$.

Proof. As Fernandez and Gray has shown in [1982], to show that a $G_{2}$-structure $\rho$ is parallel it suffices to prove that $d \rho=d^{*} \rho=0$. Clearly, $d \rho=0$, because

$$
d \rho=3 t^{2} d \omega \wedge d t+3 t^{2} d t \wedge d \omega=0 .
$$

On the other hand, $*(\omega \wedge d t)=\frac{1}{2} t^{2} \omega^{2}$, and $* d \omega=-3 d t \wedge I(d \omega)$, where $*$ is taken with respect to the cone metric on $C(M)$. This is clear, because $(\omega, \Omega)$ defines an $S U(3)$-structure on $M$, and $d \omega=3 \lambda \operatorname{Re} \Omega$. Then

$$
* \rho=\frac{3}{2} t^{4} \omega^{2}-3 t^{3} d t \wedge I(d \omega) .
$$

Since $d \Omega=-2 \sqrt{-1} \lambda \omega^{2}$ and $3 \lambda d \operatorname{Re} \Omega=d^{2} \omega=0$, we obtain $d \operatorname{Im} \Omega=-2 \lambda \omega^{2}$. This gives $d I(d \omega)=-2 \omega^{2}$, because $\lambda I(d \omega)=\operatorname{Im} \Omega$. Then (4-7) implies

$$
d(* \rho)=6 t^{3} d t \wedge \omega^{2}+3 t^{3} d t \wedge d I(d \omega)=6 t^{3} d t \wedge \omega^{2}-6 t^{3} d t \wedge \omega^{2}=0 .
$$

We proved that $C(M)$ is a parallel $G_{2}$-manifold. The converse statement is straightforward. 
In Section 2B, it is shown that the holonomy of $C(M)$ is strictly $G_{2}$, unless it is flat (in the latter case, $M$ is locally isometric to a sphere). Therefore, Proposition 4.5 implies the following corollary.

Corollary 4.6. In the assumptions of Proposition 4.5, the almost complex structure is uniquely determined by the metric, unless $M$ is locally isometric to a 6-sphere.

4D. Near Kählerness obtained from $G_{2}$-geometry. Now we can conclude the proof of Theorem 4.2.

Proof of (iii) from (ii). Let $M$ be a 6-manifold satisfying the assumptions of Theorem 4.2 (ii). Consider the cone $C(M)$ equipped with a parallel $G_{2}$-structure $\rho$ as in Proposition 4.5. Let $g_{0}$ be a cone metric on $C(M)$. From the argument used to prove Proposition 4.5, it is clear that $g_{0}$ is a metric induced by the 3 -form $\rho$ as in (2-1).

Consider the map $C(M) \stackrel{\tau}{\rightarrow} M \times \mathbb{R}$ induced by $(m, t) \mapsto(m, \log t)$, and let $g_{1}=\tau^{*} g_{\pi}$ be induced by the product metric $g_{\pi}$ on $M \times \mathbb{R}$. Denote by $\nabla_{0}, \nabla_{1}$ the corresponding Levi-Civita connections. We know that $\nabla_{0}(\rho)=0$, and we need to show that

$$
\nabla_{1}(\omega)=d \omega
$$

The metrics $g_{0}, g_{1}$ are proportional: $g_{1}=g_{0} e^{-t}$. This allows one to relate the Levi-Civita connections $\nabla_{1}$ and $\nabla_{0}$ (see for example [Ornea and Piccinni 1998]) by

$$
\nabla_{1}=\nabla_{0}+\frac{1}{2} A
$$

where $A: T M \rightarrow \operatorname{End}\left(\Lambda^{1}(M)\right)$ is an $\operatorname{End}\left(\Lambda^{1}(M)\right)$-valued 1-form mapping $X \in T M$ to

$$
(\theta, X) \operatorname{Id}-X \otimes \theta+X^{\sharp} \otimes \theta^{\sharp},
$$

with $\theta$ the 1-form defined by $\nabla_{0}\left(g_{1}\right)=g_{1} \otimes \theta, X \otimes \theta$ the tensor product of $X$ and $\theta$ considered as an endomorphism of $\Lambda^{1}(M)$, and $X^{\sharp} \otimes \theta^{\sharp}$ the dual endomorphism.

From (4-9) and $\nabla_{0}(\rho)=0$ we obtain

$$
\left.\left.\left(\nabla_{1}\right)_{X}(\rho)=(X, \theta) \rho-(\rho\lrcorner X\right) \wedge \theta+(\rho\lrcorner \theta^{\sharp}\right) \wedge X^{\sharp} .
$$

Since $\theta=\frac{d t}{t}$, we have $\nabla_{1}(\theta)=0$, and $\nabla_{1}$ preserves the decomposition $\Lambda^{*}(C(M)) \cong$ $\Lambda^{*}(M) \oplus d t \wedge \Lambda^{*}(M)$. Restricting ourselves to the $d t \wedge \Lambda^{*}(M)$-summand of this decomposition and applying (4-10), we find

$$
\left.\left(\nabla_{1}\right)_{X}\left(t^{3} \omega \wedge \theta\right)=t^{3}(d \omega\lrcorner X\right) \wedge \theta
$$

for any $X$ orthogonal to $d t$. Since $g_{1}$ is a product metric on $C(M) \cong M \times \mathbb{R}$, this leads to $\nabla \omega=d \omega$, where $\nabla$ is the Levi-Civita connection on $M$. This implies 
(4-8). So we deduced Theorem 4.2 (iii) from (ii) and the proof of Theorem 4.2 is finished.

Using Corollary 4.6, we also obtain the following useful proposition.

Proposition 4.7. Let $(M, I, g)$ be a nearly Kähler manifold. Then the almost complex structure is uniquely determined by the Riemannian structure, unless $M$ is locally isometric to a 6-sphere.

\section{Almost complex structures on 6-manifolds and their infinitesimal variations}

5A. Hitchin functional and the volume functional. Let $(M, I)$ be an almost complex 6-manifold with nowhere degenerate Nijenhuis tensor $N$, and $\mathrm{Vol}_{I}=\operatorname{det} N^{*} \otimes$ $\overline{\operatorname{det} N^{*}}$ the corresponding volume form as in (1-2). In this section we study the extrema of the functional

$$
\stackrel{\Psi}{\longrightarrow} \int_{M} \mathrm{Vol}_{I}
$$

A similar functional was studied by N. Hitchin for 6- and 7-manifolds equipped with a stable 3-form [Hitchin 2001]. Since then, this functional has acquired a pivotal role in string theory and M-theory, under the name "Hitchin functional".

Our first step is to describe the variation of $\Psi$. We denote by $\mathfrak{M}$ the space of all almost complex structures with nowhere degenerate Nijenhuis tensor on $M$.

Let $(M, I, \omega)$ be an almost complex manifold with nowhere degenerate Nijenhuis tensor

$$
N \in \operatorname{Hom}\left(\Lambda^{2} T^{1,0}(M), T^{0,1}(M)\right),
$$

$\delta \in T_{I} \mathfrak{M}$ an infinitesimal variation of $I$, and

$$
N_{\delta} \in \operatorname{Hom}\left(\Lambda^{2} T^{1,0}(M), T^{0,1}(M)\right)
$$

the corresponding variation of the Nijenhuis tensor. Consider the form

$$
\rho:=\omega(N(x, y), z)
$$

associated with the Hermitian structure on $M$ as in Theorem 1.4. After rescaling $\omega$, we assume that

$$
|\rho|_{\omega}=1
$$

Since the Nijenhuis tensor is nowhere degenerate, $\rho$ is also nowhere degenerate. Therefore, $\rho$ can be used to identify $T^{0,1}(M)$ and $\Lambda^{2} T^{1,0}(M)$, and we may consider $N_{\delta}$ as an endomorphism of $\Lambda^{0,1}(M)$. Notice that this identification maps $N$ to the identity automorphism of $\Lambda^{0,1}(M)$. 
Claim 5.1. With these assumptions,

$$
\frac{d \Psi}{d I}(\delta)=2 \operatorname{Re} \int_{M} \operatorname{Tr} N_{\delta} \operatorname{Vol}_{I}
$$

Proof. It is well known that

$$
\frac{d(\operatorname{det} A)}{d t}=\operatorname{det} A \operatorname{Tr}\left(A^{-1} \frac{d A}{d t}\right)
$$

for any matrix $A$. Applying that to the map

$$
N^{*} \otimes \bar{N}^{*}: \Lambda^{1,0}(M) \otimes \Lambda^{0,1}(M) \rightarrow \Lambda^{0,2}(M) \otimes \Lambda^{2,0}(M),
$$

we obtain that

$$
\frac{d\left(\operatorname{det}\left(N^{*} \otimes \bar{N}^{*}\right)\right)}{d I}(\delta)=\operatorname{Tr}\left(\frac{N_{\delta}^{*} \otimes \bar{N}^{*}+N^{*} \otimes \bar{N}_{\delta}^{*}}{N^{*} \otimes \bar{N}^{*}}\right) \cdot \operatorname{det}\left(N^{*} \otimes \bar{N}^{*}\right) .
$$

However, after we identify $\Lambda^{1,0}(M)$ and $\Lambda^{0,2}(M)$ as above, $N$ becomes an identity, and (5-2) gives

$$
\frac{d\left(\operatorname{det}\left(N^{*} \otimes \bar{N}^{*}\right)\right)}{d I}(\delta)=2 \operatorname{Re} \operatorname{Tr} N_{\delta} \operatorname{Vol}_{I} .
$$

Remark 5.2. We find that the extrema of the functional $\Psi(M, I)=\int_{M} \mathrm{Vol}_{I}$ are precisely those almost complex structures for which $\operatorname{Re} \operatorname{Tr} N_{\delta}=0$ for any infinitesimal variation $\delta$ of $I$.

5B. Variations of almost complex structures and the Nijenhuis tenor. It is convenient, following Kodaira and Spencer, to consider infinitesimal variantions of almost complex structures as tensors $\delta \in \Lambda^{0,1}(M) \otimes T^{1,0}(M)$. Indeed, a complex structure on a vector space $V, \operatorname{dim}_{\mathbb{R}} V=2 d$, can be considered as a point of the Grassmannian of $d$-dimensional planes in $V \otimes \mathbb{C}$. The tangent space to a Grassmannian at a point $W \subset V \otimes \mathbb{C}$ is given by $\operatorname{Hom}(W, V \otimes \mathbb{C} / W)$.

Consider the $(0,1)$-part $\nabla^{0,1}$ of the Levi-Civita connection

$$
\nabla^{0,1} \delta \in \Lambda^{0,1}(M) \otimes T^{1,0}(M) \otimes \Lambda^{0,1}(M),
$$

and let

$$
\bar{\partial}: \Lambda^{0,1}(M) \otimes T^{1,0}(M) \rightarrow \Lambda^{0,2}(M) \otimes T^{1,0}(M)
$$

denote the composition of $\nabla^{0,1}$ with the exterior multiplication map

$$
\Lambda^{0,1}(M) \otimes T^{1,0}(M) \otimes \Lambda^{0,1}(M) \rightarrow \Lambda^{0,2}(M) \otimes T^{1,0}(M) .
$$

The following claim is well known. 
Claim 5.3. Let $(M, I)$ be an almost complex manifold, and

$$
\delta \in \Lambda^{0,1}(M) \otimes T^{1,0}(M)
$$

an infinitesimal variation of almost complex structure. Denote by $N_{\delta} \subset \Lambda^{2,0}(M) \otimes$ $T^{0,1}(M)$ the corresponding infinitesimal variation of the Nijenhuis tensor (see Section $5 A$ ). Then $\bar{N}_{\delta}=\bar{\partial} \delta$, where $\bar{\partial}$ is the differential operator defined above.

Proof. The proof follows from a direct computation; see for example [Kodaira and Spencer 1958].

Claim 5.3 can be used to study the deformation properties of the functional $I \stackrel{\Psi}{\longrightarrow} \int_{M} \mathrm{Vol}_{I}$ constructed above; see Section 5A. Indeed, from Remark 5.2 it follows that $\Psi$ has an extremum at $I$ if and only if $\operatorname{Re} \operatorname{Tr} N_{\delta}=0$ for any $\delta \in$ $\Lambda^{0,1}(M) \otimes T^{1,0}(M)$. Using the identification $T^{1,0}(M) \cong \Lambda^{2,0}(M)$, provided by the nondegenerate $(3,0)$-form as above, we can consider $\delta$ as a $(2,1)$-form on $M$. Then

$$
\bar{\partial} \delta \in \Lambda^{0,2}(M) \otimes \Lambda^{2,0}(M)=\Lambda^{2,2}(M)
$$

is the (2,2)-part of $d \delta$. Under these identifications, and using $|\rho|_{\omega}=1$ from (5-1), we can express $\operatorname{Tr} \bar{N}_{\delta}$ as

$$
\operatorname{Tr} \bar{N}_{\delta}=\frac{\bar{\partial} \delta \wedge \omega}{\operatorname{Vol}_{I}}
$$

where $\bar{\partial}$ is a $(0,1)$-part of the de Rham differential. This gives the following claim.

Claim 5.4. Let $(M, I, \omega)$ be an almost complex Hermitian 6-manifold with nowhere degenerate Nijenhuis tensor. Assume that the corresponding 3-form $\rho$ satisfies $|\rho|_{\omega}=1$; see (5-1). Consider the functional $\Psi(I)=\int_{M} \mathrm{Vol}_{I}$ on the space of such almost complex structures. Then

$$
\frac{d \Psi}{d I}(\delta)=2 \operatorname{Re} \int_{M} \bar{\partial} \delta \wedge \omega
$$

where $\delta \in \Lambda^{0,1}(M) \otimes T^{1,0}(M)$ is an infinitesimal deformation of an almost complex structure $I$, considered as a $(2,1)$-form on $M$.

Proof. The claim is implied immediately by (5-3) and Claim 5.1.

Comparing Claim 5.4 with Remark 5.2, we find the following corollary.

Corollary 5.5. In assumptions of Claim 5.4, I is an extremum of $\Psi$ if and only if

$$
\operatorname{Re} \int_{M} \bar{\partial} \delta \wedge \omega=0
$$

for any $\delta \in \Lambda^{2,1}(M)$. 
Integrating by parts, we find that (5-4) is equivalent to

$$
\operatorname{Re} \int_{M} \delta \wedge \bar{\partial} \omega=0
$$

and to $\bar{\partial} \omega=0$. This gives the following proposition.

Proposition 5.6. Let $(M, I, \omega)$ be an almost complex Hermitian 6-manifold with nowhere degenerate Nijenhuis tensor. Consider the functional $\Psi(I)=\int_{M} \mathrm{Vol}_{I}$ on the space of such almost complex structures on $M$. Then $I$ is an extremum of $\Psi$ if and only if $d \omega$ lies in $\Lambda^{3,0}(M) \oplus \Lambda^{0,3}(M)$.

Now, Proposition 5.6 together with Theorem 4.2 implies Theorem 2.2. Notice that by Corollary 3.3, the nearly Kähler Hermitian structure on $(M, I)$ is (up to a constant multiplier) uniquely determined by $I$.

\section{Acknowledgments}

I am grateful to Robert Bryant, Nigel Hitchin, Paul-Andi Nagy and Uwe Semmelmann for valuable advices and consultations. P.-A. Nagy also suggested adding Proposition 4.7. Much gratitude to the referee for useful suggestions and the invaluable help of finding a multitude of minor errors.

\section{References}

[Acharya and Gukov 2004] B. S. Acharya and S. Gukov, "M theory and singularities of exceptional holonomy manifolds", Phys. Rep. 392:3 (2004), 121-189. MR 2004k:53072 arXiv hep-th/0409191

[Agricola and Friedrich 2004] I. Agricola and T. Friedrich, "On the holonomy of connections with skew-symmetric torsion”, Math. Ann. 328:4 (2004), 711-748. MR 2005f:53072 Zbl 1055.53031 arXiv math.DG/0305069

[Atiyah and Witten 2002] M. Atiyah and E. Witten, " $M$-theory dynamics on a manifold of $G_{2}$ holonomy”, Adv. Theor. Math. Phys. 6:1 (2002), 1-106. MR 2004f:53046 Zbl 1033.81065 arXiv hepth/0107177

[Baum et al. 1991] H. Baum, T. Friedrich, R. Grunewald, and I. Kath, Twistors and Killing spinors on Riemannian manifolds, Teubner-Texte zur Mathematik 124, B. G. Teubner Verlagsgesellschaft, Stuttgart, 1991. MR 94a:53077 Zbl 0734.53003

[Bismut 1989] J.-M. Bismut, "A local index theorem for non-Kähler manifolds", Math. Ann. 284:4 (1989), 681-699. MR 91i:58140 Zbl 0666.58042

[Bryant 2000] R. Bryant, "Pseudoholomorphic Bundles over Almost Hermitian 6-Manifolds", 2000. Talk at the International Congress on Differential Geometry in memory of Alfred Gray (Bilbao, 2000).

[Bryant 2006] R. L. Bryant, "On the geometry of almost complex 6-manifolds", Asian J. Math. 10:3 (2006), 561-605. MR 2007g:53029 Zbl 1114.53026 arXiv math.DG/0508428

[Butruille 2005] J.-B. Butruille, "Classification des variétés approximativement kähleriennes homogènes”, Ann. Global Anal. Geom. 27:3 (2005), 201-225. MR 2006f:53060 Zbl 1079.53044 arXiv math.DG/0401152 
[Cleyton and Swann 2004] R. Cleyton and A. Swann, "Einstein metrics via intrinsic or parallel torsion", Math. Z. 247:3 (2004), 513-528. MR 2005i:53054 Zbl 1069.53041 arXiv math.DG/0211446 [Dragomir and Ornea 1998] S. Dragomir and L. Ornea, Locally conformal Kähler geometry, Progress in Mathematics 155, Birkhäuser, Boston, 1998. MR 99a:53081 Zbl 0887.53001

[Eells and Salamon 1985] J. Eells and S. Salamon, "Twistorial construction of harmonic maps of surfaces into four-manifolds”, Ann. Scuola Norm. Sup. Pisa Cl. Sci. (4) 12:4 (1985), 589-640 (1986). MR 87i:58042 Zbl 0627.58019

[Fernández and Gray 1982] M. Fernández and A. Gray, "Riemannian manifolds with structure group G2”, Ann. Mat. Pura Appl. (4) 132 (1982), 19-45 (1983). MR 84e:53056 Zbl 0524.53023

[Friedrich 2003] T. Friedrich, "On types of non-integrable geometries", pp. 99-113 in Proceedings of the 22nd Winter School "Geometry and Physics" (Srní, Czech Republic, 2002), 2003. MR 2004e:53039 Zbl 1079.53041 arXiv math.DG/0205149

[Friedrich and Ivanov 2002] T. Friedrich and S. Ivanov, "Parallel spinors and connections with skew-symmetric torsion in string theory”, Asian J. Math. 6:2 (2002), 303-335. MR 2003m:53070 Zbl pre01925761 arXiv math.DG/0102142

[Gauduchon 1984] P. Gauduchon, "La 1-forme de torsion d'une variété hermitienne compacte", Math. Ann. 267:4 (1984), 495-518. MR 87a:53101 Zbl 0523.53059

[Gauduchon 1995] P. Gauduchon, "Structures de Weyl-Einstein, espaces de twisteurs et variétés de type $S^{1} \times S^{3}$ ”, J. Reine Angew. Math. 469 (1995), 1-50. MR 97d:53048 Zbl 0858.53039

[Gini et al. 2005] R. Gini, L. Ornea, and M. Parton, "Locally conformal Kähler reduction", J. Reine Angew. Math. 581 (2005), 1-21. MR MR2132669 (2006c:53077) Zbl 1080.53066 arXiv math.DG/ 0208208

[Gray 1965] A. Gray, "Minimal varieties and almost Hermitian submanifolds", Michigan Math. J. 12 (1965), 273-287. MR 32 \#1658 Zbl 0132.16702

[Gray 1970] A. Gray, "Nearly Kähler manifolds", J. Differential Geometry 4 (1970), 283-309. MR 42 \#2404 Zbl 0201.54401

[Gray 1971] A. Gray, "Weak holonomy groups", Math. Z. 123 (1971), 290-300. MR 45 \#2614 Zbl 0222.53043

[Gray 1976] A. Gray, "The structure of nearly Kähler manifolds”, Math. Ann. 223:3 (1976), 233248. MR 54 \#6010 Zbl 0345.53019

[Grunewald 1990] R. Grunewald, "Six-dimensional Riemannian manifolds with a real Killing spinor”, Ann. Global Anal. Geom. 8:1 (1990), 43-59. MR 92a:58146 Zbl 0704.53050

[Hitchin 1981] N. Hitchin, "Kählerian twistor spaces", Proc. London Math. Soc. (3) 43:1 (1981), 133-150. MR 84b:32014 Zbl 0474.14024

[Hitchin 2000] N. Hitchin, "The geometry of three-forms in six dimensions", J. Differential Geom. 55:3 (2000), 547-576. MR 2002m:53070 Zbl 1036.53042 arXiv math.DG/0010054

[Hitchin 2001] N. Hitchin, "Stable forms and special metrics", pp. 70-89 in Global differential geometry: the mathematical legacy of Alfred Gray (Bilbao, 2000), edited by M. Fernández and J. A. Wolf, Contemp. Math. 288, Amer. Math. Soc., Providence, RI, 2001. MR 2003f:53065 Zbl 1004.53034 arXiv math.DG/0107101

[Ivanov 2004] S. Ivanov, "Connections with torsion, parallel spinors and geometry of Spin(7) manifolds”, Math. Res. Lett. 11:2-3 (2004), 171-186. MR 2005e:53071 Zbl 1073.53065 arXiv math.DG/0111216

[Ivanov et al. 2006] S. Ivanov, M. Parton, and P. Piccinni, "Locally conformal parallel $G_{2}$ and Spin(7) manifolds", Math. Res. Lett. 13:2-3 (2006), 167-177. MR 2007e:53057 Zbl 1118.53028 arXiv math/0509038 
[Joyce 2000] D. D. Joyce, Compact manifolds with special holonomy, Oxford Mathematical Monographs, Oxford University Press, Oxford, 2000. MR 2001k:53093 Zbl 1027.53052

[Kamishima and Ornea 2005] Y. Kamishima and L. Ornea, "Geometric flow on compact locally conformally Kähler manifolds”, Tohoku Math. J. (2) 57:2 (2005), 201-221. MR 2006g:53112 Zbl 1083.53068 arXiv math.DG/0105040

[Kiričenko 1977] V. F. Kiričenko, “K-spaces of maximal rank”, Mat. Zametki 22:4 (1977), 465-476. English translation: Math. Notes 22: 4 (1978) 751-757. MR 57 \#13756 Zbl 0414.53018

[Kodaira and Spencer 1958] K. Kodaira and D. C. Spencer, "On deformations of complex analytic structures. I, II”, Ann. of Math. (2) 67 (1958), 328-466. MR 22 \#3009 Zbl 0128.16901

[Moroianu et al. 2005] A. Moroianu, P.-A. Nagy, and U. Semmelmann, "Unit Killing vector fields on nearly Kähler manifolds", Internat. J. Math. 16:3 (2005), 281-301. MR 2006e:53056 Zbl 1077. 53038 arXiv math.DG/0406492

[Muškarov 1987] O. Muškarov, "Structures presque hermitiennes sur des espaces twistoriels et leurs types”, C. R. Acad. Sci. Paris Sér. I Math. 305:7 (1987), 307-309. MR 89b:53068 Zbl 0626.53028

[Nagy 2002a] P.-A. Nagy, "Nearly Kähler geometry and Riemannian foliations”, Asian J. Math. 6:3 (2002), 481-504. MR 2003m:53043 Zbl 1041.53021 arXiv math.DG/0203038

[Nagy 2002b] P.-A. Nagy, “On nearly-Kähler geometry”, Ann. Global Anal. Geom. 22:2 (2002), 167-178. MR 2003g:53073 Zbl 1020.53030 arXiv math.DG/0110065

[Ornea and Piccinni 1998] L. Ornea and P. Piccinni, "Weyl structures on quaternionic manifolds", pp. 231-236 in Quaternionic structures in mathematics and physics (Trieste, 1994), edited by G. Gentili et al., SISSA, Trieste, 1998. MR 99k:53098 Zbl 0946.53019 arXiv math.DG/0105041

[Verbitskiı̌ 2004] M. S. Verbitskiŭ, "Theorems on the vanishing of cohomology for locally conformally hyper-Kähler manifolds”, Tr. Mat. Inst. Steklova 246 (2004), 64-91. MR 2005h:53071 Zbl 1101.53027 arXiv math.DG/0302219

Received September 13, 2007. Revised November 1, 2007.

\section{Misha VERBITSKY}

INSTITUTE OF THEORETICAL AND EXPERIMENTAL PHYSICS

B. CHEREMUSHKINSKAYA, 25

Moscow, 117259

RUSSIA

verbit@mccme.ru 